\title{
Histone variant macroH2A1 deletion in mice causes female-specific steatosis
}

\author{
Mathieu Boulard ${ }^{1,2 \dagger}$, Sébastien Storck ${ }^{1,2 \dagger}$, Rong Cong ${ }^{1,2,3}$, Rodrigo Pinto ${ }^{1,2}$, Hélène Delage ${ }^{1,2}$, Philippe Bouvet ${ }^{1,2^{*}}$
}

\begin{abstract}
Background: Vertebrate heterochromatin contains a non-allelic variant of the histone $\mathrm{H} 2 \mathrm{~A}$ called macroH2A1, which has the characteristic of being three times the size of the canonical H2A. The macroH2A1 C-terminal extension can recruit onto chromatin the poly-ADP-ribose polymerase (PARP)1, which is crucial for DNA repair. This led to the speculation that macroH2A1 could be essential for genome surveillance; however, no experimental evidence supported this hypothesis. Because macroH2A1 has been found to be enriched on the inactive $X$-chromosome in females, it is thought to play a role in sex chromosome dosage compensation through its ability to regulate gene expression. However, more genetic data are needed to further understand the function of macroH2A1 in mammals.

Results: Deletion of the murine gene H2afy, which encodes for macroH2A1, resulted in lipid accumulation in liver. Hepatic steatosis caused by H2afy disruption occurred specifically in homozygous mutant females. The metabolic disorder constantly affected half of the number of homozygote females. Given the mixed genetic background of the mutants, an unreported genetic modifier is likely to influence the penetrance of the phenotype. In addition, the X-linked thyroxine-binding globulin (Tbg) gene was specifically upregulated in steatotic livers. Chromatin immunoprecitation indicated that macroH2A 1 is enriched at the Tbg promoter in wild-type female animals, indicating that increased Tog expression in H2afy null mutants is likely to be a direct consequence of the absence of macroH2A1. Furthermore, male mice, which are not prone to the metabolic disorder, had a reduced level of macroH2A1 incorporated into the Tbg promoter.

Conclusions: Because TBG is the main carrier of the thyroid hormone T4, which regulates energy metabolism, we propose that overexpression of TBG is responsible for the fat accumulation observed in H2afy-deficient liver. Moreover, our results suggest that the sexual dimorphism of the steatotic phenotype is probably due to the different incorporation of macroH2A1 in males and females. In combination with previous studies, our data demonstrate a role for macroH2A1 in regulating homeostasis in a sex-dependent manner, subject to genetic background.
\end{abstract}

\section{Background}

The histone variant macroH2A1 has the ability to substitute for H2A within the nucleosome and to establish chromatin domains with specific properties, due to its C-terminal non-histone macrodomain [1]. Incorporation of macroH2A1 results in the addition of a C-terminal domain of $25 \mathrm{kDa}$ to a histone fold motif, and thus constitutes the largest known nucleosome modification [1]. MacroH2A1 dynamic recruitment onto the inactive

\footnotetext{
* Correspondence: pbouvet@ens-lyon.fr

† Contributed equally

'Université de Lyon, Ecole Normale Supérieure de Lyon, Laboratoire JoliotCurie (CNRS USR 3010), 46 allée d'Italie, 69364 Lyon cedex 07, France
}

$\mathrm{X}$-chromosome $\left(\mathrm{X}_{\mathrm{i}}\right)$ upon inactivation in female mammals indicates a possible function in sex-chromosome dosage compensation [2-5]. However, quantities of macroH2A are similar in males and females, suggesting that a role in $\mathrm{X}$ inactivation is not the sole function of this variant [6].

The presence of macroH2A 1 at promoter regions was shown to inhibit gene expression by in vitro transcription analysis of reconstituted nucleosomes containing macroH2A1 [7-9]. However, knockdown studies performed in cultured cells demonstrated that macroH2A1 is required for both transcriptional silencing [10] and induction $[11,12]$. This dual role of macroH2A1 in
C Biomed Central

C 2010 Boulard et al; licensee BioMed Central Ltd. This is an Open Access article distributed under the terms of the Creative Commons Attribution License (http://creativecommons.org/licenses/by/2.0), which permits unrestricted use, distribution, and reproduction in any medium, provided the original work is properly cited. 
transcription was investigated further in a recent study, which showed that $12 \%$ of the genes enriched in macroH2A1 escape silencing [13]. Furthermore, the same study showed that a subset of genes is downregulated upon macroH2A1 knockdown, indicating that macroH2A1 could act as a positive regulator of transcription at some loci [13].

In vivo, the presence of macroH2A1 is essential for the silencing of a class of endogenous (non-ecotropic) murine leukaemia viruses [14]. In addition, macroH2A1 plays a function in $\mathrm{NAD}^{+}$catabolism through its role in the two main pathways of $\mathrm{NAD}^{+}$consumption. First, macroH2A1 binds to poly-ADP polymerase (PARP)-1 and inhibits its enzymatic activity $[12,15,16]$. PARP- 1 is a nuclear enzyme that catalyzes the cleavage of $\mathrm{NAD}^{+}$ into ADP-ribose and nicotinamide, and the subsequent transfer of ADP-ribose units to target proteins [17]. PARP-1 is implicated in chromatin structure modulation, transcription regulation and double-strand break (DSB) DNA repair [17]. Secondly, macroH2A1 binds the metabolite $O$-acetyl-ADP-ribose produced by the $\mathrm{NAD}^{+}$ dependent histone deacetylase Sirt1 [18]. Although all these recent findings provide biochemical evidence indicating that macroH2A 1 plays a role in $\mathrm{NAD}^{+}$catabolism, genetic evidence is needed to identify its function in energy metabolism in mammals.

In this study, we generated loss-of-function animals through targeted disruption of H2afy, the gene that encodes macroH2A1. Mice that lacked macroH2A1 displayed no obvious visible phenotype and no increased sensitivity to ionizing radiation, which excludes an essential function for macroH2A1 in genome surveillance. However, we found that macroH2A1-deficient females exhibit liver lipid homeostasis defects that lead to hepatic steatosis. Interestingly, lipid accumulation in the liver was specific to females. We provide evidence of reactivation of the $\mathrm{X}$-linked thyroxine-binding globulin $(T b g)$ gene specifically in steatotic livers. We also demonstrate that the histone variant macroH2A1 is specifically enriched in nucleosomes that occupy the Tbg promoter in female hepatocytes, indicating that $T b g$ overexpression is a direct consequence of H2afy disruption.

\section{Results}

Targeting the mouse H2afy locus

We generated an H2afy null-mutant mouse strain by homologous recombination in ES cells. Our strategy resulted in the complete disruption of exon 2, which encodes the translation start codon and approximately half of the histone domain (Figure 1a, b).

Mating of heterozygous animals resulted in offspring of all genotypes at the mendelian ratio (95 heterozygous, 68 wild types and 48 homozygous) and the overall sex ratio was close to 1:1. Mice homozygous for the H2afy mutation were viable, and survived to adulthood without discernible morphological abnormalities. Both male and female $H 2 a f^{-1-}$ mutants were fertile and produced litter sizes comparable with the wild type.

Absence of the macroH2A1 protein in tissues was confirmed in homozygous mice by immunoblotting using a specific antibody (Figure 1c). To determine whether compensation might be involved, the expression level of macroH2A2 (H2afy2) was examined in different tissues. MacroH2A2, which is $80 \%$ identical to macroH2A1, is encoded by a separate gene located on chromosome $10[19,20]$. Little is known about this second macroH2A subtype, but it was recently shown to be crucial for zebrafish (Danio rerio) embryogenesis [21]. In contrast to many human cells and mouse, zebrafish embryos predominantly express $H 2 a f y 2$, whereas $H 2 a f y$ was not detectable [21].

No overexpression of H2afy 2 was observed in the absence of macroH2A1 (Figure 1c). Therefore, it is unlikely that the absence of a phenotype in H2afy-deficient animals is the result of a functional compensation by H2afy2.

\section{MacroH2A1 is not essential for DNA damage repair}

Because macroH2A1 interacts with PARP-1, which is essential for early DNA damage recognition and base excision repair by sensing DNA nicks [17], it has been proposed that macroH2A1 could play a role in the process of DNA repair through its ability to recruit PARP1 onto chromatin $[12,16]$.

To determine whether macroH2A1 is essential for DSB repair in vivo, we tested the sensitivity of H2afydeficient mice to whole-body ionizing sublethal irradiation. Mice lacking macroH2A1 did not show any increased sensitivity to gamma radiation compared with control littermates (Figure 2). Histological sections through the duodenum did not show any difference in the size of villi between mutant and control mice (data not shown), indicating normal ionizing radiationinduced cell renewal. We conclude that macroH2A1 is not required for accurate DNA break repair in vivo.

\section{Absence of macroH2A1 induces hepatic steatosis in females}

Histological examination of all major organs, including brain, kidney, spleen, heart, visceral fat and reproductive organs, showed no differences between macroH2A1deficient mice and control littermates up to 12 weeks of age. However, analysis of liver from $\mathrm{H} 2 a \mathrm{fy}^{-1-}$ females revealed signs of hepatic steatosis (Figure 3, Table 1). Of 17 mutant females, nine displayed periportal hepatocellular macrovacuolation (Figure 3a, b), a phenotype that was not observed in any mutant male or in wild-type 


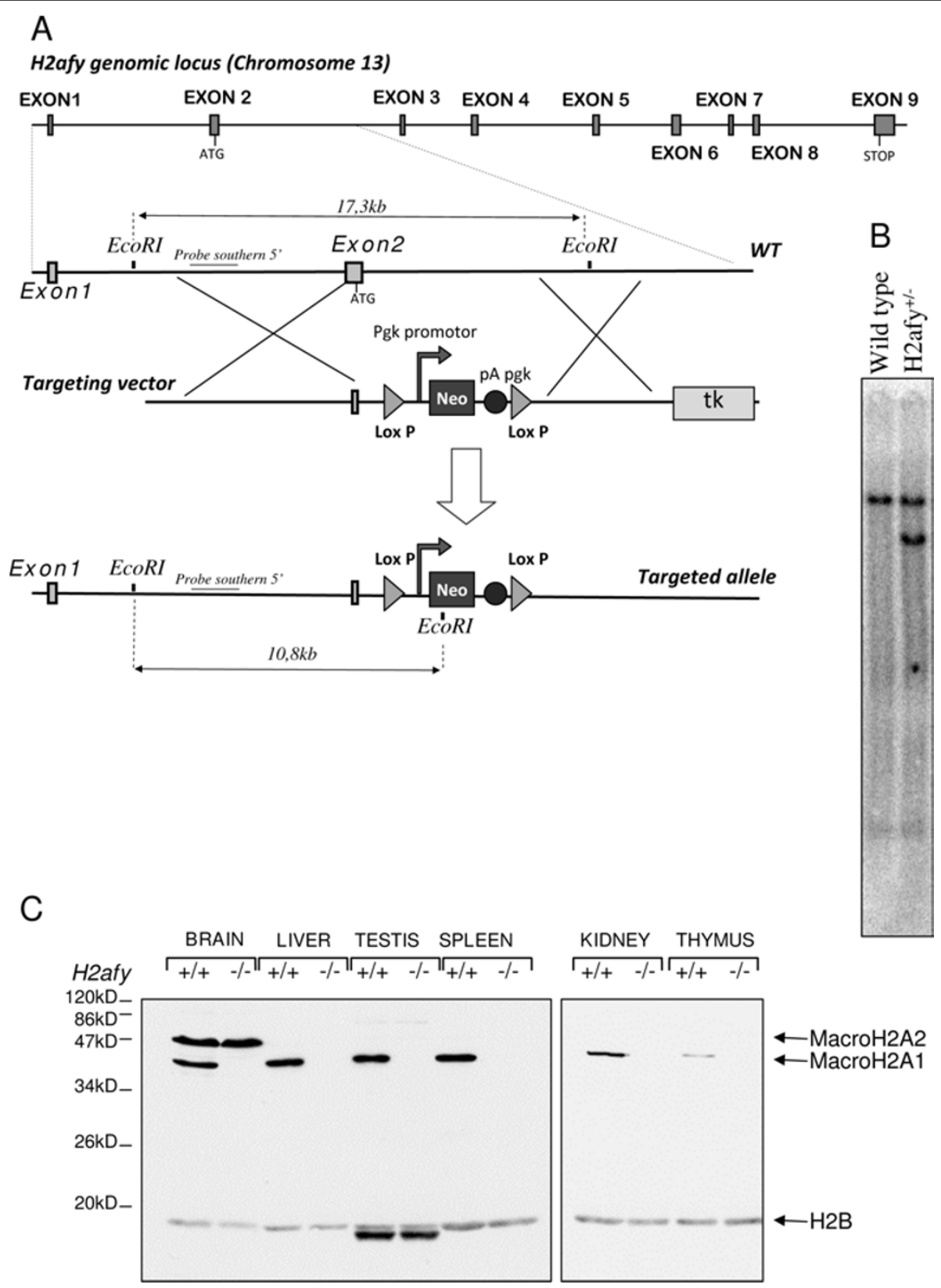

Figure 1 H2afy gene-targeting strategy and genotyping of cells and mice. (a) Schematic diagrams of the H2afy locus, the targeting vector and the mutant Hzafy alleles are shown. The exons are numbered. The targeting construct containing a neomycin (Neo) cassette flanked by two loxP sites (triangles) was inserted in place of exon 2, resulting in the deletion of the first coding exon, which encodes amino acids 1 to 57. The targeted locus lacks the translation initiation codon and half of the sequence coding for the histone region, and should therefore function as a null allele. The first 18 nucleotides (non-coding) of exon 2 were not modified by the targeting in order to maintain the correct splicing of the first exon to the second. This mutation affects both splicing isoforms macroH2A1.1 and macroH2A1.2, because they are generated by alternative splicing of exon 6 . Probes used for the Southern blot studies and relevant restriction fragments predicted by digestion of the recombinant are shown. The sizes of the diagnostic fragments for the wild-type (WT) and Hzafy mutant alleles are indicated. (b) Southern blot analysis of recombinant embryonic stem cells (digested with EcoRI). The blots were hybridized with the probes shown in (a). (c) Immunodetection of macroH2A1 and macroH2A2 in H2afy ${ }^{-1-}$ and wild-type mice. Nuclear extracts were prepared from various tissues. The protein macroH2A1 was undetectable in the mutant tissue, proving that the targeted mice were null mutants. There was no overexpression of macroH2A2, which was detected only in brain and embryo (data not shown). H2B shows equal protein loading between all lanes. The additional fast migrating bands seen in testis are probably caused by the crossreactivity of the anti-H2B antibody with the testis-specific variant of histone $\mathrm{H} 2 \mathrm{~B}$. 


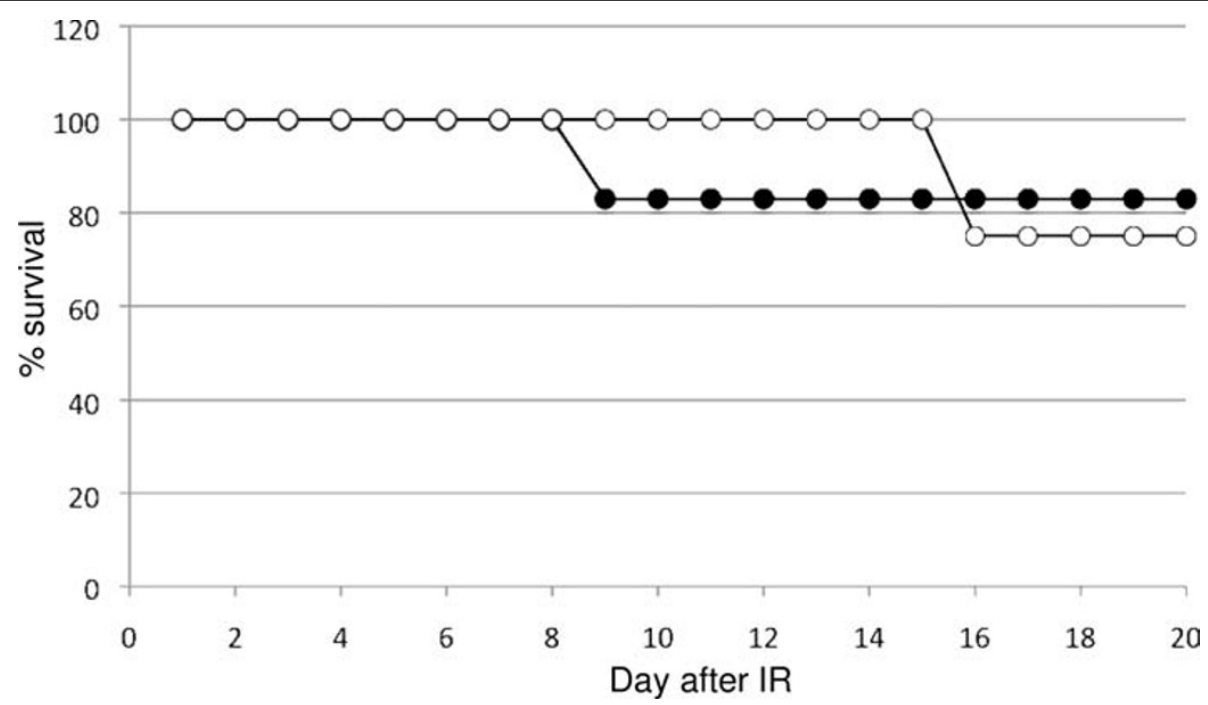

Figure 2 MacroH2A1 is not required for DNA repair in vivo. Survival of 7-week-old H2afy ${ }^{-1}$ mice and littermate controls exposed to 6.5 Gy whole-body gamma irradiation. Four H2afy- (black circles) and six control mice were used (open circles). H2afy-deficient mice were not hypersensitive to DNA damage induced by ionizing irradiation, suggesting that macroH2A1 is not essential for DNA repair.

male and female mice. We found by staining tissue sections with oil red $\mathrm{O}$ that this vacuolation was the result of abnormal triglyceride accumulation in liver (Figure $3 c, d)$. Hence, we conclude that the absence of macroH2A1 induces steatosis.

\section{Reactivation of non-ecotropic MLV is not the cause of hepatic steatosis}

Incorporation of macroH2A1 in chromatin has been shown to be required for the silencing of the endogenous retrovirus murine leukemia virus (MLV) [14]. To explore a possible correlation between MLV overexpression and hepatic steatosis, we assessed MLV env transcription in $\mathrm{H}_{2} \mathrm{afy}^{-1-}$ female steatotic livers compared with $\mathrm{H} 2 a \mathrm{fy}^{-1-}$ female healthy livers and wild-type female livers (Figure 4a).

Transcription of MLV elements was not detected above background level in three of four wild-type samples. Surprisingly, an MLV transcript was detected in one wild-type sample (Figure 4a), showing that MLV can also be transcribed in the presence of macroH $2 \mathrm{~A} 1$; this may reflect the segregation of a gene unlinked to H2afy that regulates MLV expression. Quantification of the northern-blot data confirmed that MLV silencing is lost in the absence of macroH2A1, as previously reported [14]. MLV expression level, normalized to GAPDH, was upregulated by 2.3 -fold in $\mathrm{H}_{2} \mathrm{afy}^{-1-}$ mutants (Figure 4b), but no significant difference was observed between healthy mutant livers and steatotic livers (Figure 4b). We therefore exclude a relationship between hepatic steatosis and MLV overexpression in the absence of macroH2A1.

\section{Hepatic accumulation of lipids is correlated with upregulation of the X-linked Tbg gene}

A major cause of hepatic steatosis is the increased fatty acid flux to the liver caused by a high level of free fatty acids in plasma [22]. To gain further insight into the molecular causes of the triglyceride accumulation in the absence of macroH2A1, we investigated a possible correlation between this phenotype and the expression level of a few genes important for lipid metabolism that have been previously shown to be regulated by macroH2A1 [23] (Figure 5).

The expression level of lipoprotein lipase $(L p l)$, which catalyses the hydrolysis of the triacylglycerol component of circulating chylomicrons and very low-density lipoproteins [24], was not affected by the mutation (Figure $5 b)$. Mice lacking macroH2A1 presented an upregulation of CD36 (Figure 5a), a fatty acid transporter in muscle and adipose tissue. By contrast, Thrsp (thyroid hormone responsive SPOT14 homolog), a regulator of de novo lipogenesis, was downregulated in $\mathrm{H}_{2} \mathrm{afy}^{-1-}$ livers compared with controls (Figure 5c) [25]. Thus, our results suggest that Thrsp belongs to the recently discovered category of genes that are positively regulated by macroH2A1 [13].

No differences in Thrsp or CD36 expression were observed between $\mathrm{H} 2 \mathrm{afy}^{-/}$females showing a steatotic liver and those with a healthy liver (Figure 5a, c). Therefore, it is unlikely that the steatotic phenotype observed in $\mathrm{H}_{2} \mathrm{afy} \mathrm{y}^{-1-}$ females was a consequence of the deregulation of CD36 and Thrsp expression.

Remarkably, the X-linked Tbg gene (also named Serpina7) was overexpressed by sixfold in $\mathrm{H}_{2} a \mathrm{fy}^{-/-}$female 


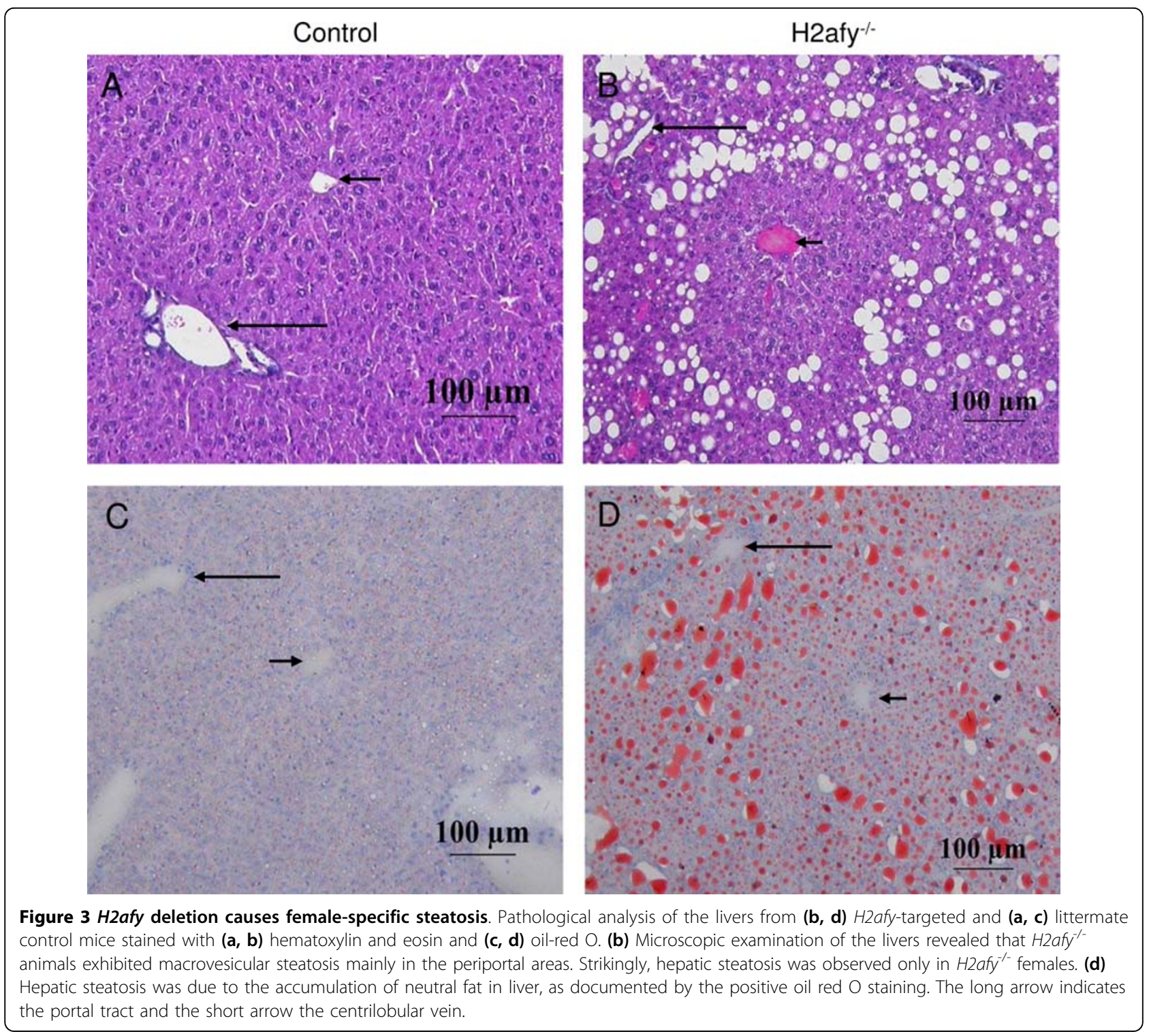

Table 1 Hepatic steatosis incidence

\begin{tabular}{lllll}
\hline & \multicolumn{2}{l}{$\begin{array}{l}\text { Control } \\
\text { littermates }\end{array}$} & \multicolumn{2}{l}{ macroH2A $^{-/-}$} \\
\cline { 2 - 6 } & Male & Female & Male & Female \\
\hline Mice presenting hepatic steatosis, $\mathrm{n}$ & 0 & 0 & 0 & 9 \\
\hline Total examined mice, $\mathrm{n}$ & 3 & 7 & 8 & 17 \\
\hline Incidence, $\%$ & 0 & 0 & 0 & 53 \\
\hline
\end{tabular}

mice with a steatotic phenotype compared with healthy mutant or wild-type females, which both expressed $T b g$ at the same level (Figure 5e). TBG is a thyroid hormone-binding protein, which carries the majority of the hormone T4 in the serum [26]. Given that thyroid hormones influence general energy metabolism and specifically promote increases in lipid metabolism and counteract lipid accumulation [27], overexpression of $\mathrm{Tbg}$ in $\mathrm{H} 2 \mathrm{afy}^{-/-}$females and its consequences on lipid metabolism could be the origin of the hepatic steatosis we observed.

To test if the overexpression of $T b g$ was a consequence of a global reactivation of $X_{i}$ in the absence of macroH2A1, we examined expression of $D l g 3$, another gene located on the $\mathrm{X}$-chromosome (Figure $5 \mathrm{~d}$ ). Although the expression level of $D \lg 3$ was slightly elevated in mutant mice, $D l g 3$ did not show any significant upregulation in steatotic liver (Figure 5d). Together, these data indicate that the inactive $\mathrm{X}$-chromosome is not fully reactivated in the absence of macroH2A1, indicating that macroH2A 1 is not required for global silencing of $\mathrm{X}_{\mathrm{i}}$. 


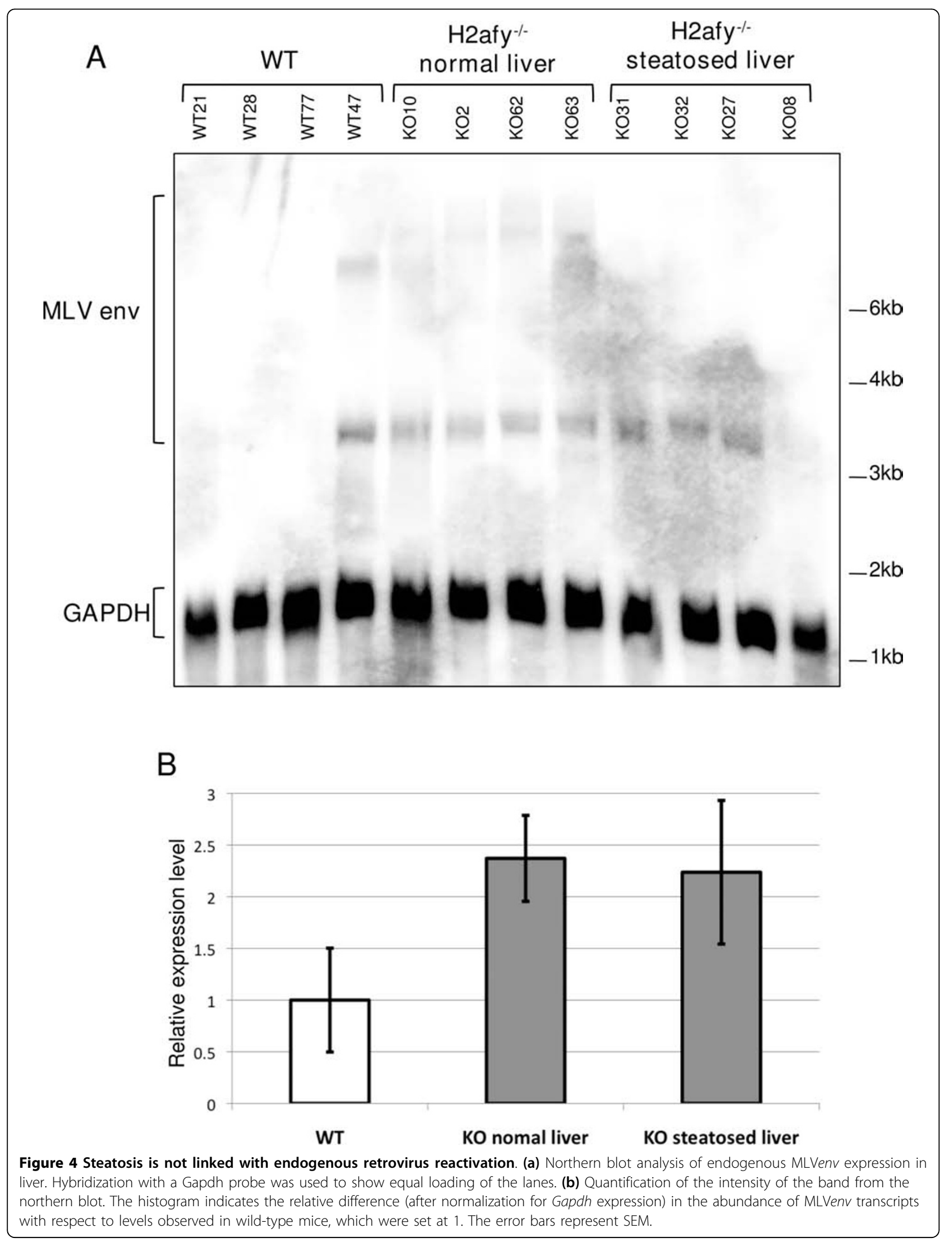




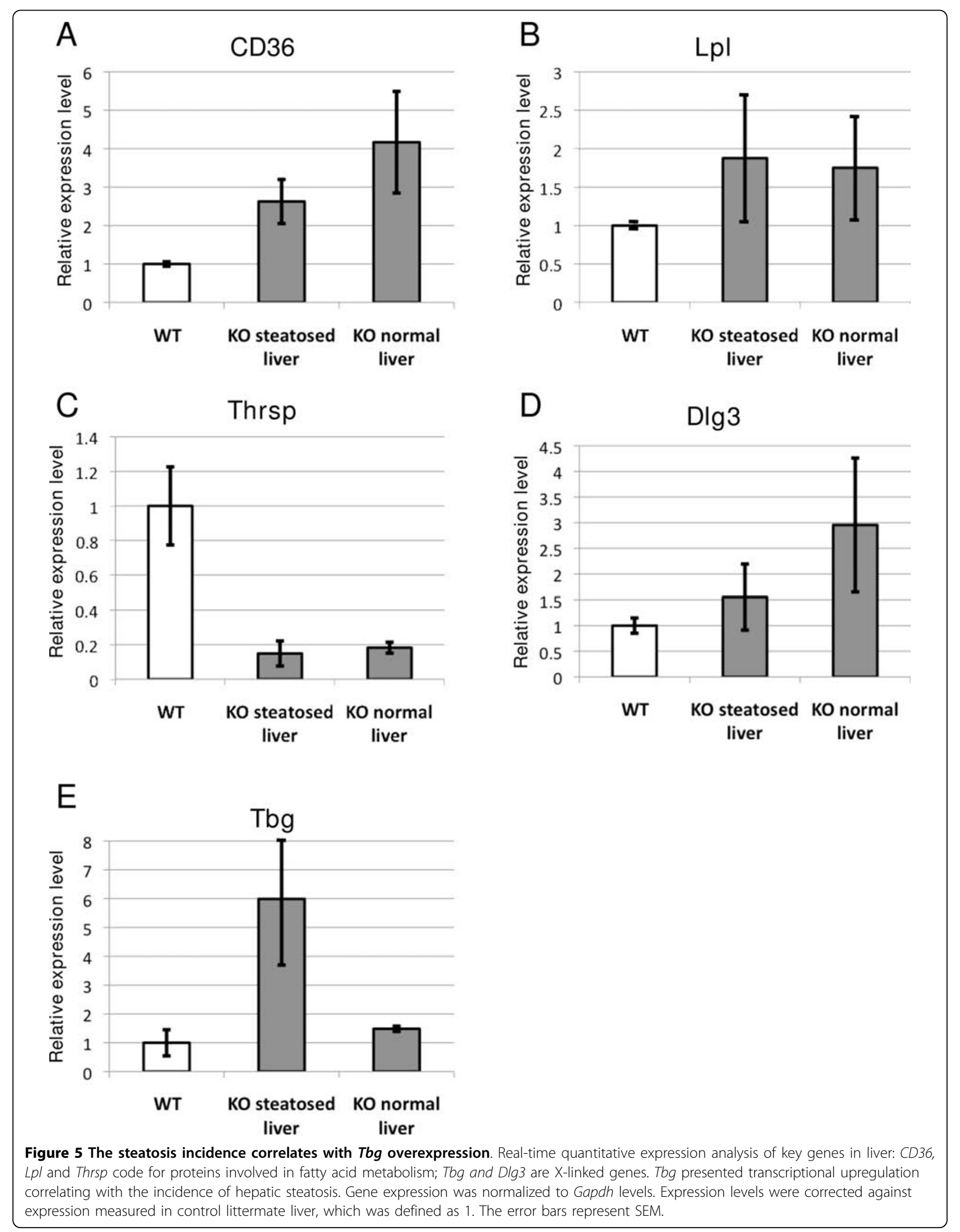




\section{MacroH2A1 occupies the female but not the male Tbg promoter}

To determine if macroH2A1 directly controls the expression of genes most perturbed in $\mathrm{H}_{2} a \mathrm{fy}^{-/-}$liver, we performed chromatin immunoprecipitation (ChIP) experiments to test for the presence of macroH2A1 in nucleosomes that occupied promoters of those genes in livers of wild-type animals (Figure 6).

We first analysed chromatin extracted from female livers (Figure 6a). We did not detect the presence of macroH $2 \mathrm{~A} 1$ above the background level on the $L p l$ gene, which was not deregulated in the absence of macroH2A1 (Figure 5b). By contrast, wild-type female hepatocytes displayed high levels of macroH2A1 incorporated into the sequence upstream of the $T b g$ transcription start site (Figure 6a). This finding strongly suggests that increased expression of $T b g$ measured in $\mathrm{H}_{2} \mathrm{afy}^{-1-}$ livers is a direct consequence of H2afy disruption.

To determine the molecular cause of the sexual dimorphism of the steatosis promoted by H2afy disruption, we compared macroH2A1 levels on the Tbg promoter in male versus female animals (Figure 6b). Consistent with our first analysis (Figure 6a), we found that macroH2A1 is 10 -fold enriched on the Tbg promoter in females (Figure 6b). Furthermore, our ChIP analysis shows that in contrast to females, the Tbg promoter in males has low amounts of bound macroH2A1 (Figure $6 \mathrm{~b})$. It should be mentioned that in a previous study [23], this sex-specific difference was not noted. This might be the result of differences in the purification method of macroH2A containing nucleosomes (immunoprecipitation in this study; biochemical purification in the previous study [23]). However, in the previous study, Tbg expression was shown to be four times higher in the livers of wild-type adult males than in wild-type adult females [23]. Hence, the sexual dimorphic expression of $T b g$ in wild-type mice is probably caused by the low incorporation of macroH2A1 on the $T b g$ promoter in male hepatocytes.

We conclude that the sexual dimorphism of the phenotype caused by H2afy deletion is caused by the sexdependent incorporation of macroH2A 1 at specific loci, as evidenced by the sex-specific incorporation of macroH2A1 on the Tbg promoter.

\section{Discussion}

Our study provides novel insights into macroH2A1 functions in X-chromosome inactivation and lipid metabolism. Loss of function of H2afy causes hepatic accumulation of lipid specifically in female mice.

Interestingly, a recent report has demonstrated that mice fed with a methyl-deficient diet that induces hepatic steatosis had an elevated level of macroH2A [28].
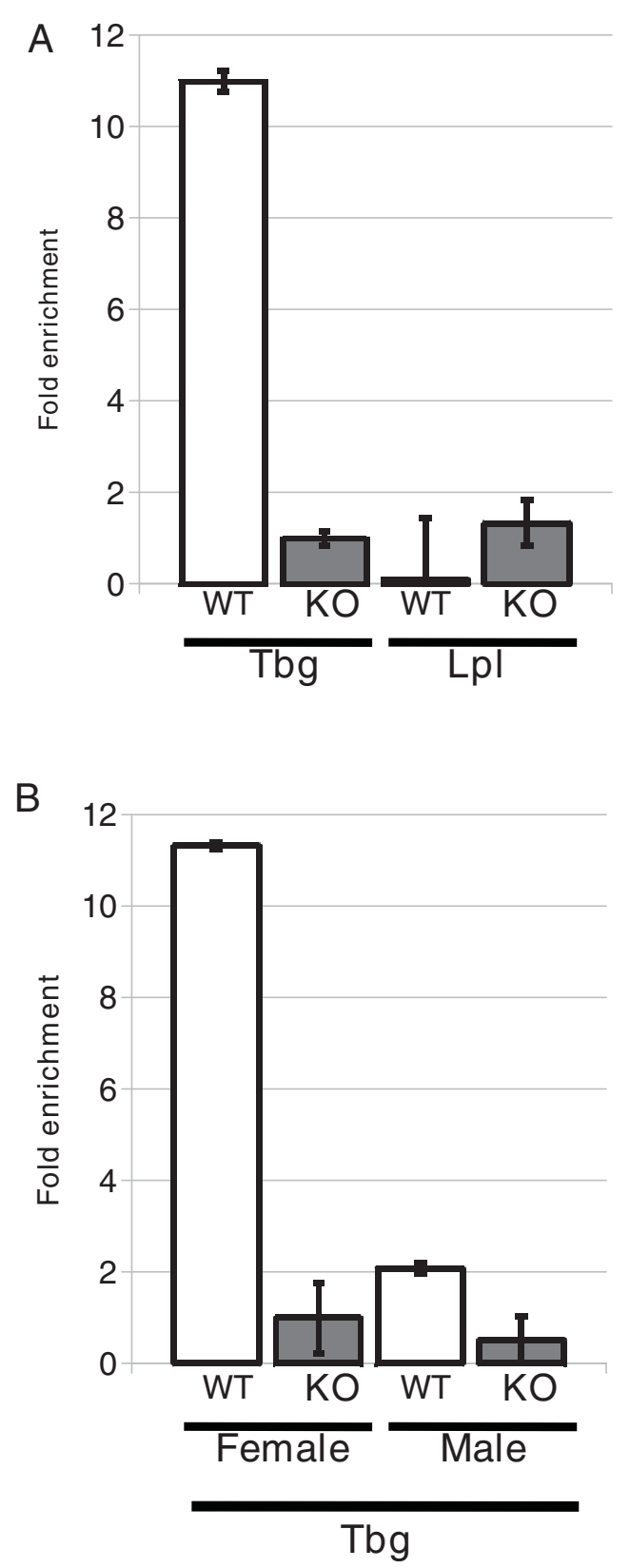

Figure 6 MacroH2A1 is specifically incorporated into the female $\mathrm{Tbg}$ promoter. Chromatin immunoprecipitation (ChIP) analysis of the association of macroH2A 1 with key gene promoters involved in lipid metabolism. (a) ChIP analysis of macroH2A1 enrichment level on Tbg and $L p /$ promoters in females. The graph shows that macroH2A1 was enriched at Tbg promoter but not at the $L p /$ promoter in female hepatocytes. (b) The Tbg promoter in male mice was not significantly occupied by macroH2A1. ChIP using liver chromatin from wild-type (WT) animals and Hzafy-1knockout (KO) mice was performed using anti-macroH2A1 antibody. Quantitative PCR was then performed with specific primers for promoter regions of the Tbg and $L p /$ genes as indicated in Materials and methods. The results are presented as the fold enrichment in wild-type animals compared with Tbg promoter DNA amplified in $\mathrm{KO}$ female mice. The graph represents the average of three independent experiments. 
Although this seemingly contradicts our results, we propose that the elevated level of macroH2A reported in this study reflects a homeostatic response within the liver that is ultimately saturated after prolonged exposure to high levels of lipids. In the light of this study [28], our findings indicate that macroH2A could be incorporated at high levels in chromatin in order to circumscribe hepatic lipid accumulation.

Using a similar approach, Changolkar and colleagues have independently shown that H2afy-deficient mice are viable, and that the mutation does not result in any obvious macroscopic phenotype [23]. Those independent data consistently demonstrated that macroH2A 1 does not play a fundamental role during development; however, two recent studies have shown that macroH2A1 is preferentially incorporated at developmental gene promoters $[13,21]$. This apparent contradiction might be explained by the potential existence of redundant chromatin silencing pathways. One hypothesis suggests functional compensation by the polycomb repressive complex (PRC)2, as indicated by its colocalisation with macroH2A1 [29]. This hypothesis is also sustained by the genome-wide colocalisation between macroH2A1 and the trimethylation of lysine 27 of histone $\mathrm{H} 3$, the histone posttranslational modification catalysed by PRC2 $[13,30]$. However, the possible redundant roles of PRC2 and macroH2A1 remain to be experimentally tested.

In addition, the study of Changolkar et al. showed that H2afy disruption has a minor influence on gene expression, as only seven genes displayed significant abnormal expression in the absence of macroH2A1 [23]. The surprisingly low number of genes affected by the macroH2A1 deletion could be explained either by possible functional compensation by another redundant chromatin repressive mechanism or by the presence of genetic modifiers, as discussed below. Very interestingly, four genes deregulated in $\mathrm{H} 2 \mathrm{afy}^{-/-}$livers encode proteins directly involved in fatty acid metabolism, which further supports the role of macroH2A1 in lipid homeostasis control as highlighted in our study. We have independently confirmed the abnormal upregulation of three of these genes in $\mathrm{H}_{2} \mathrm{afy}^{-1-}$ liver. However, we found a decreased level of Thrsp in mutant female liver, whereas the study of Changolkar et al. concluded that Thrsp expression is not affected by H2afy disruption in female hepatocytes [23].

The major discordance between these two parallel studies concerns liver steatosis; there is no mention of this in the study from Changolkar et al. [23]. This important difference could be explained by the different genetic backgrounds of mice in these two studies. The study by Changolkar et al. was performed using a fixed mutation on the C57Bl6 background whereas the mutants we analysed had a genetic background composed of half 129Ola and half C57Bl6 (intercrosses of the F2 generation). Such interference of the genetic background with the phenotype has been extensively documented for many genes [31]. Likewise, variation of the genetic background has been reported to have significant influences on both the quantities of macroH2A1 in steatotic liver and the incidence of steatosis [28].

Additionally, our data reveal that the penetrance of the steatotic phenotype of female progenies of $\mathrm{C} 57 \mathrm{Bl} /$ $6 \times 129$ Ola mice was very close to half. This consistent penetrance in half the animals over several intercross generations indicates the existence of a genetic modifier present in one of the two mixed genetic backgrounds that is able to modify the penetrance of the phenotype resulting from $H 2$ afy deletion. Identification of this genetic modifier should expand our knowledge of the metabolic pathway controlled by macroH2A 1 in female.

Our study uncovers part of the molecular mechanism by which macroH2A1 regulates lipid homeostasis. Indeed, we show that macroH2A1 silences $\mathrm{Tbg}$ expression in female liver, as evidenced by the overexpression of $\mathrm{Tbg}$ in the absence of macroH2A1. We also provide evidence of specific enrichment of macroH2A1 on the $\mathrm{Tbg}$ promoter, which indicates that macroH2A1 directly regulates $T b g$ transcription.

The consequences of $T b g$ overexpression in mice are unknown. However, studies in patients with amplification of the $T b g$ gene revealed that excess TBG can lead to hypothyroidism and altered metabolism [32,33]. Thus, we propose that elevated levels of TBG, the main T4 hormone carrier, could be the cause of the alteration in lipid metabolism responsible for hepatic steatosis in the absence of macroH2A1.

The reactivation of the X-linked $T b g$ caused by H2afy disruption raises the possibility that the remarkable sexual dimorphism of the phenotype might be due to $X_{i}$ silencing failure in the absence of macroH2A1. However, we found that expression of $D \lg 3$, another X-linked gene, is not affected by $H 2 a f y$ disruption. Therefore, $\mathrm{X}_{\mathbf{i}}$ is not entirely reactivated in the absence of macroH2A1. In good agreement with our conclusion, a global analysis of gene expression in $H 2 a f^{-1-}$ liver did not report evidence of $X_{i}$ reactivation in the absence of macroH2A1 [23]. This notion was reinforced by a previous study, which reported that $H 2 a f y$ knockdown is not sufficient to reactivate the transcription of a reporter gene stably inserted in $\mathrm{X}_{\mathrm{i}}$ [34].

Given the low level of macroH2A1 incorporated into the male $T b g$ promoter, we propose that the sexual dimorphism of the phenotype could be due to the selective incorporation of macroH2A 1 on the $T b g$ promoter in female cells. Our measurements show that the amount of macroH2A 1 incorporated into the $T b g$ 
promoter is about fivefold higher in female mice than in male mice. This 5:1 ratio cannot be explained by the twofold difference of the X-chromosome copy number between the sexes. Instead, the difference in macroH2A1 occupancy at the same locus between males and females is probably caused by an unbalanced incorporation of macroH2A1 between the sexes. Specific enrichment on the female X-linked $\mathrm{Tbg}$ promoter could possibly be a reflection of the specific incorporation of macroH2A1 on $\mathrm{X}_{\mathrm{i}}[2-4,35]$. The different levels of macroH2A1 on the $T b g$ promoter locus between the sexes might also be a consequence of a more general differential genomic localisation of histone variants between different cell types (for example, male versus female).

\section{Conclusions}

Although much effort has been devoted to understanding the role of macroH2A1 in gene silencing, its biological function remains elusive. Recent advances that uncovered its association with PARP-1 have led to the speculation that macroH2A1 could be involved in DNA repair. However, we report here that $H 2 a f y^{-1-}$ mutants are not hypersensitive to DNA DSB, excluding a fundamental role of macroH2A1 in the control of the DNA damage response.

In addition, we have shown that loss of function of H2afy causes hepatic accumulation of lipid specifically in females. We also report a correlation between macroH2A1 occupancy on the Tbg promoter and its specific overexpression in steatotic liver, which strongly supports our hypothesis that $\mathrm{Tbg}$ deregulation is the major cause of lipid metabolism dysfunction in the absence of macroH2A1. Thus, our study provides novel insights into macroH2A1 functions in X-linked gene silencing and lipid metabolism.

The importance of chromatin in the control of homeostasis has recently begun to be uncovered with the discovery of the role of histone deacetylase and histone demethylase in the regulation of energy metabolism $[36,37]$. Our work highlights that histone variants are also involved in the regulation of lipid metabolism.

\section{Methods}

All experiments on mice were carried out with CREA Rhône-Alpes (France) committee approval. Mice were normally housed in cages with littermates of the same sex. Animals were kept in a 12-hour light/dark cycle and fed standard diet rodent chow (SAFE \#A04-10 and SAFE \#A03-10 for breeders; Perotech Sciences Inc., Toronto, ON, Canada).

\section{Generation of macroH2A1 targeted mice}

H2afy was disrupted by replacing exon 2 with a neomycin resistance cassette. Both recombination fragments were first amplified using DNA polymerase (Phusion; New England BioLabs, Ipswich, MA, USA) and appropriate primers (Table 2) on DNA extracted from E14.1 ES cells, then cloned into SalI and $\mathrm{XhoI}$ restriction sites, respectively, of the pLN-TK targeting vector (Figure 1). The resulting vector was linearized with $M l u \mathrm{I}$ and electroporated into E14.1 ES cells (129/Ola strain) as described previoulsy [38]. After antibiotic selection (G418/Geneticin; Gibco-BRL, Grand Island, NY, USA), homologous recombinants were identified by PCR using the set of primers named 'screen2 F' and 'NeoLOXP2 R' shown in Table 2. Homologous recombination was confirmed by Southern blotting and extensive restriction analysis, using flanking and internal probes (data not shown). Two different ES clones heterozygous for the targeted mutation were injected into $\mathrm{C} 57 \mathrm{Bl} / 6$ blastocysts and transferred into pseudopregnant foster mothers. One clone gave high-level chimeras and transmitted the disrupted $H 2 a f y$ allele to offspring at high frequency when crossed into $\mathrm{C} 57 \mathrm{Bl} / 6$ mice. The mice were maintained by intercrosses of the original $129 \mathrm{Ola} \times \mathrm{C} 57 \mathrm{Bl} / 6$ mice. The animals were genotyped by PCR on genomic DNA

Table 2 List of primers

\begin{tabular}{lll}
\hline Primer name & $\mathbf{5}^{\prime} \boldsymbol{\rightarrow} \mathbf{3}^{\prime}$ & \\
\cline { 2 - 3 } & Forward & Reverse \\
\hline $5^{\prime \prime}$ recombination arm & AGCTCGAGCTAGAATGGTGAACAGGTTGGAAGG & TTGTCGACAAACAGATCAGCGAGCTCACTGC \\
\hline $3^{\prime}$ recombination arm & AGCTCGAGCTGGTCTGCACACATCCTTAAGG & ACAGGAGTCAAGATGGTGGCTGGG \\
\hline Dlg3 $^{*}$ & CTATGGGACCAGCATCCAGT & TGTGCTTGCTGCAGTCTCT \\
\hline Tbg $^{*}$ & AAGGCTGTGCTACACATTGG & CGGATAACAGGGTGAGAGG \\
\hline Lpl $^{*}$ & GAGCGAGAACATTCCCTTCA & TCTGAGTCTCTCCGGCTTC \\
\hline CD36* $^{*}$ & GCAAAGTTGCATAATTGAGTCC & GTCTGTGCCATTAATCATGTCG \\
\hline Thrsp $^{*}$ & GAGTGCATCTGTGGACTTGG & GAGTAACTGCGACATGACACC \\
\hline GAPDH* $^{*}$ & CAACTACATGGTCTACATGTC & CGCCAGTAGACTCCACGAC \\
\hline $185^{*}$ & CGGCTACCACATCCAAGGAA & GCTGGAATTACCGCGGCT \\
\hline
\end{tabular}

*Reverse transcriptase PCR. 
isolated from finger biopsy, using the primer pairs 'screen F' located in intron 1-2 and 'screen R' located in the targeted portion of intron 2-3, and a third primer complementary to the neo gene 'NeoLOXP2 R' (Table 2). This resulted in the amplification of a $500 \mathrm{bp}$ fragment from the wild-type macroH $2 A 1$ allele and a $310 \mathrm{bp}$ fragment from the null allele.

\section{Immunoblotting}

Nuclear proteins were isolated from tissues in accordance with the protocol described previously [39]. Briefly, $10 \mu \mathrm{g}$ of nuclear proteins were separated by SDS-PAGE and transferred onto nitrocellulose membranes. For the detection of macroH2A1, a polyclonal antibody (Millipore Inc., Billericay, MA, USA) was used. MacroH2A2 was detected using a rabbit polyclonal antibody (cat. no. ab4173; Abcam Inc., Cambridge, MA, USA) at a dilution of 1:2000. Histone $\mathrm{H} 2 \mathrm{~B}$ was detected using a rabbit polyclonal antibody (cat. no. 07-371; Millipore) at a dilution of 1:5000 [39].

\section{Southern blotting}

For Southern blotting, $10 \mu \mathrm{g}$ of DNA were digested with $100 \mathrm{U}$ of EcoRI, then separated by agarose gel electrophoresis and transferred onto a nitrocellulose membrane, followed by UV crosslinking. Filters were hybridized with a ${ }^{32} \mathrm{P}$-labelled probe located 5 ' to the sequences in the targeting vector (Figure 1). Membranes were washed at $65^{\circ} \mathrm{C}$, twice with $1 \times$ saline sodium citrate (SSC) buffer plus $0.1 \%$ SDS for 15 minutes each, and twice with $0.1 \times$ SSC plus $1 \%$ SDS for 30 minutes each, then exposed to a phosphor screen for 24-72 hours. The gel images were acquired with a scanner (Fujifilm 217 FLA-5100; Fujifilm Holdings Corp., Tokyo, Japan) and the pictures were analyzed with Multigauge V.3.0 software (Fujifilm).

\section{Survival of mice after whole body gamma irradiation}

For induction of DNA damage, 7-8-week-old H2afy ${ }^{-1-}$ mice and control littermates were exposed to wholebody sublethal ionizing irradiation. Animals were irradiated with a ${ }^{137} \mathrm{Cs}$ source at a rate of $0.68 \mathrm{~Gy} / \mathrm{min}$ in single doses of $6.5 \mathrm{~Gy}$. H2afy-deficient mice and seven control littermates were irradiated, and survival was determined. Four days after irradiation, one mutant and one wild type were killed for histopathological analysis of the small intestine.

\section{Histological analysis}

Formaldehyde-fixed, paraffin wax-embedded sections were prepared from liver samples, and were stained with hematoxylin and eosin using standard methods. Frozen liver sections were stained with oil red O. Sections were photographed at $20 \times$ magnification.

\section{Total RNA preparation, reverse transcription and} quantitative PCR

Total RNA was isolated using TRIzol reagent (Invitrogen Corp., Carlsbad, CA, USA) and treated with DNaseI, then $5 \mu \mathrm{g}$ of RNA were reverse transcribed (RevertAid Reverse Transcriptase; Fermentas, Burlington, ON, Canada). Quantitative reverse transcriptase (RT) PCR reactions were performed using the Light-Cycler system (Roche Applied Science; Indianapolis, IN, USA) to determine mRNA levels of Tbg, Lpl, CD36, Thrsp and Dlg3 (specific primers are listed in Table 2). All cDNAs were normalized using primers to Gapdh. Expression levels were corrected against expression measured in control littermate liver, which was defined as 1 . cDNA levels from wild-type $(\mathrm{n}=2)$, steatotic macroH $2 A 1^{-1-}$ $(\mathrm{n}=4)$ and healthy macroH $2 A 1^{-1-}(\mathrm{n}=3)$ were compared, and results expressed as mean \pm SEM.

\section{Northern blotting}

For Northern blotting, $10 \mu \mathrm{g}$ of total RNA were denatured and subjected to electrophoresis in a $1 \%$ agarose plus $1.9 \%$ formaldehyde gel in $1 \times$ MOPS buffer, and transferred to a nitrocellulose membrane. The membrane was hybridized with probes derived from radiolabeled extension of random hexamers annealed to the purified $1011 \mathrm{bp}$ fragments produced by MelARVenv digestion by NcoI (described in [40]) or Gapdh previously amplified from cDNA using the primers listed in Table 2. Quantification of the intensity of the bands was performed using MultiGauge V.3.0 software (Fujifilm). Gapdh mRNA served as an internal control, and expression levels of MLV were set to 1 for the wild-type littermate controls. Results are expressed as mean \pm SEM.

\section{Chromatin immunoprecipitation}

Mouse liver tissue ( $1 \mathrm{~g}$ ) was homogenized in buffer $\mathrm{H}$ (250 mM sucrose, $3 \mathrm{mM} \mathrm{CaCl}_{2}$ in $20 \mathrm{mM}$ Tris $\mathrm{pH} 7.4$ ) in the presence of a cocktail of protease inhibitors cocktail (Roche Applied Science, Basel, Switzerland) and spun in a centrifuge at $280 \mathrm{~g}$, for $10 \mathrm{~min}$ at $4^{\circ} \mathrm{C}$ (A-4-81 rotor; Eppendorf North America, Westbury, NY, USA). The resulting pellet was collected and washed three times in phosphate-buffered saline (PBS) and carefully resuspended in a 2.4 M sucrose cushion (2.4 M sucrose, $1 \mathrm{mM}$ $\mathrm{MgCl} 2,10 \mathrm{mM}$ Tris $\mathrm{pH} 6.8$ and protease inhibitor cocktail). The suspension was centrifuged at 168,000 $\mathrm{g}$ for 3 hours at $4^{\circ} \mathrm{C}$ (SW41 Ti rotor; Beckmann-Coulter Inc., Brea, CA, USA). The nuclear fraction was carefully collected and washed three times in PBS plus protease inhibitor cocktail, then counted and divided into aliquots. Following this, $1.5 \times 10^{6}$ nuclei were crosslinked for 10 min in $1 \%$ paraformaldehyde at room temperature and sheared by sonication to produce DNA fragments averaging $500 \mathrm{bp}$ in length. The sheared chromatins were 
immunoprecipitated with anti-macroH2A1 antibody (cat. no. 07-219; Upstate Biotech). Precipitated DNA and protein complexes were uncrosslinked and purified by phenol/choloroform extraction. Purified DNA was used as templates for qPCR quantification using $\mathrm{Tbg}$ or $\mathrm{Lpl}$ specific primers listed in Table 2.

\section{Acknowledgements}

This work was supported by grants from CNRS, ANR (NT05-1_41978), Association pour la Recherche contre le Cancer (ARC n³901) and Région Rhone-Alpes (MIRA 2008). MB was supported in part by ARC predoctoral fellowship. We thank the Plateau de Biologie Expérimentale de la Souris (ENS, Lyon) for assistance in the generation and the breeding of mutant mice, and the Institut Clinique de la Souris, Strasbourg, D Watrelot-Virieux and C Thirion-Delalande for histological analyses. We are grateful to $\mathrm{TH}$ Bestor for helpful comments on the manuscript.

\section{Author details}

'Université de Lyon, Ecole Normale Supérieure de Lyon, Laboratoire JoliotCurie (CNRS USR 3010), 46 allée d'Italie, 69364 Lyon cedex 07, France. ${ }^{2}$ Université de Lyon, Ecole Normale Supérieure de Lyon, Laboratoire de Biologie Moléculaire de la Cellule, 46 allée d'Italie, 69364 Lyon cedex 07, France. ${ }^{3}$ The Institute of Biomedical Sciences and School of Life Sciences, East China Normal University, Shanghai 200241, China.

\section{Authors' contributions}

$M B, S S, R C, R P$ and $P B$ conceived of and designed the experiments. MB, SS, $R C, R P$ and $H D$ performed the experiments. $M B, S S, R C, R P, H D$ and $P B$ analysed the data. MB and SS generated the Hzafy-targeted mouse strain. $\mathrm{MB}$, SS and PB coordinated the work and co-wrote the manuscript. All authors read and approved the final manuscript.

\section{Competing interests}

The authors declare that they have no competing interests.

Received: 1 December 2009 Accepted: 1 April 2010

Published: 1 April 2010

\section{References}

1. Pehrson JR, Fried VA: MacroH2A, a core histone containing a large nonhistone region. Science 1992, 257:1398-1400.

2. Costanzi C, Pehrson JR: Histone macroH2A1 is concentrated in the inactive X chromosome of female mammals. Nature 1998, 393:599-601.

3. Mermoud JE, Costanzi C, Pehrson JR, Brockdorff N: Histone macroH2A1.2 relocates to the inactive $\mathrm{X}$ chromosome after initiation and propagation of X-inactivation. J Cell Biol 1999, 147:1399-1408.

4. Mietton F, Sengupta AK, Molla A, Picchi G, Barral S, Heliot L, Grange T, Wutz A, Dimitrov S: Weak, but uniform enrichment of the histone variant macroH2A1 along the inactive $\mathrm{X}$ chromosome. Mol Cell Biol 2008, 29:150-6.

5. Rasmussen TP, Mastrangelo MA, Eden A, Pehrson JR, Jaenisch R: Dynamic relocalization of histone MacroH2A1 from centrosomes to inactive $X$ chromosomes during X inactivation. J Cell Biol 2000, 150:1189-1198.

6. Rasmussen TP, Huang T, Mastrangelo MA, Loring J, Panning B, Jaenisch R: Messenger RNAs encoding mouse histone macroH2A1 isoforms are expressed at similar levels in male and female cells and result from alternative splicing. Nucleic Acids Res 1999, 27:3685-3689.

7. Angelov D, Molla A, Perche PY, Hans F, Cote J, Khochbin S, Bouvet $P$, Dimitrov S: The histone variant macroH2A interferes with transcription factor binding and SWI/SNF nucleosome remodeling. Mol Cell 2003, 11:1033-1041.

8. Chang EY, Ferreira H, Somers J, Nusinow DA, Owen-Hughes T, Narlikar GJ: MacroH2A allows ATP-dependent chromatin remodeling by SWI/SNF and ACF complexes but specifically reduces recruitment of SWI/SNF (dagger). Biochemistry 2008, 47:13726-32.

9. Doyen CM, An W, Angelov D, Bondarenko V, Mietton F, Studitsky VM, Hamiche A, Roeder RG, Bouvet P, Dimitrov S: Mechanism of polymerase II transcription repression by the histone variant macroH2A. Mol Cell Biol 2006, 26:1156-1164.

10. Agelopoulos M, Thanos D: Epigenetic determination of a cell-specific gene expression program by ATF- 2 and the histone variant macroH2A. EMBO J 2006, 25:4843-4853.

11. Choo JH, Kim JD, Kim J: MacroH2A1 knockdown effects on the Peg3 imprinted domain. BMC Genomics 2007, 8:479.

12. Ouararhni K, Hadj-Slimane R, Ait-Si-Ali S, Robin P, Mietton F, Harel-Bellan A, Dimitrov $S$, Hamiche A: The histone variant $\mathrm{mH} 2 \mathrm{~A} 1.1$ interferes with transcription by down-regulating PARP-1 enzymatic activity. Genes Dev 2006, 20:3324-3336.

13. Gamble MJ, Frizzell KM, Yang C, Krishnakumar R, Kraus WL: The histone variant macroH2A1 marks repressed autosomal chromatin, but protects a subset of its target genes from silencing. Genes Dev 2009, 24:21-32.

14. Changolkar LN, Singh G, Pehrson JR: macroH2A1-dependent silencing of endogenous murine leukemia viruses. Mol Cell Biol 2008, 28:2059-2065.

15. Barbera AJ, Chodaparambil JV, Kelley-Clarke B, Joukov V, Walter JC, Luger K, Kaye KM: The nucleosomal surface as a docking station for Kaposi's sarcoma herpesvirus LANA. Science 2006, 311:856-861.

16. Nusinow DA, Hernandez-Munoz I, Fazzio TG, Shah GM, Kraus WL, Panning B: Poly(ADP-ribose) polymerase 1 is inhibited by a histone H2A variant, $\mathrm{MacroH} 2 \mathrm{~A}$, and contributes to silencing of the inactive $X$ chromosome. J Biol Chem 2007, 282:12851-12859.

17. Schreiber V, Dantzer F, Ame JC, de Murcia G: Poly(ADP-ribose): novel functions for an old molecule. Nat Rev Mol Cell Biol 2006, 7:517-528.

18. Kustatscher G, Hothorn M, Pugieux C, Scheffzek K, Ladurner AG: Splicing regulates NAD metabolite binding to histone macroH2A. Nat Struct Mol Biol 2005, 12:624-625.

19. Costanzi C, Pehrson JR: MACROH2A2, a new member of the MACROH2A core histone family. J Biol Chem 2001, 276:21776-21784.

20. Chadwick BP, Willard HF: Histone $H 2 A$ variants and the inactive $X$ chromosome: identification of a second macroH2A variant. Hum Mol Genet 2001, 10:1101-1113.

21. Buschbeck M, Uribesalgo I, Wibowo I, Rue P, Martin D, Gutierrez A, Morey L, Guigo R, Lopez-Schier H, Di Croce L: The histone variant macroH2A is an epigenetic regulator of key developmental genes. Nat Struct Mol Biol 2009, 16:1074-9.

22. den Boer M, Voshol PJ, Kuipers F, Havekes LM, Romijn JA: Hepatic steatosis: a mediator of the metabolic syndrome. Lessons from animal models. Arterioscler Thromb Vasc Biol 2004, 24:644-649.

23. Changolkar LN, Costanzi C, Leu NA, Chen D, McLaughlin KJ, Pehrson JR: Developmental changes in histone macroH2A1-mediated gene regulation. Mol Cell Biol 2007, 27:2758-2764.

24. Merkel M, Eckel RH, Goldberg IJ: Lipoprotein lipase: genetics, lipid uptake, and regulation. J Lipid Res 2002, 43:1997-2006.

25. LaFave LT, Augustin LB, Mariash CN: S14: insights from knockout mice. Endocrinology 2006, 147:4044-4047.

26. Schreiber $\mathrm{G}$ : The evolutionary and integrative roles of transthyretin in thyroid hormone homeostasis. J Endocrinol 2002, 175:61-73.

27. Moreno M, de Lange P, Lombardi A, Silvestri E, Lanni A, Goglia F: Metabolic effects of thyroid hormone derivatives. Thyroid 2008, 18:239-253.

28. Pogribny IP, Tryndyak VP, Bagnyukova TV, Melnyk S, Montgomery B, Ross SA, Latendresse JR, Rusyn I, Beland FA: Hepatic epigenetic phenotype predetermines individual susceptibility to hepatic steatosis in mice fed a lipogenic methyl-deficient diet. J Hepatol 2009, 51:176-186.

29. Buschbeck M, Uribesalgo I, Wibowo I, Rue P, Martin D, Gutierrez A, Morey L, Guigo R, Lopez-Schier H, Di Croce L: The histone variant macroH2A is an epigenetic regulator of key developmental genes. Nat Struct Mol Biol 2009, 16:1074-1079.

30. Chadwick BP: Variation in Xi chromatin organization and correlation of the H3K27 me3 chromatin territories to transcribed sequences by microarray analysis. Chromosoma 2007, 116:147-157.

31. Muller $\mathrm{U}$ : Ten years of gene targeting: targeted mouse mutants, from vector design to phenotype analysis. Mech Dev 1999, 82:3-21.

32. Mori $Y$, Miura Y, Takeuchi H, Igarashi Y, Sugiura J, Saito H, Oiso Y: Gene amplification as a cause of inherited thyroxine-binding globulin excess in two Japanese families. J Clin Endocrinol Metab 1995, 80:3758-3762.

33. Refetoff $S$, Fang VS, Marshall JS: Studies on human thyroxine-binding globulin (TBG). IX. Some physical, chemical, and biological properties of radioiodinated TBG and partially desialylated TBG. J Clin Invest 1975, 56:177-187. 
34. Hernandez-Munoz I, Lund AH, Stoop van der P, Boutsma E, Muijrers I, Verhoeven E, Nusinow DA, Panning B, Marahrens Y, van Lohuizen M: Stable $\mathrm{X}$ chromosome inactivation involves the PRC1 Polycomb complex and requires histone MACROH2A1 and the CULLIN3/SPOP ubiquitin E3 ligase. Proc Natl Acad Sci USA 2005, 102:7635-7640.

35. Csankovszki G, Panning B, Bates B, Pehrson JR, Jaenisch R: Conditional deletion of Xist disrupts histone macroH2A localization but not maintenance of X inactivation. Nat Genet 1999, 22:323-324.

36. Tateishi $\mathrm{K}$, Okada Y, Kallin EM, Zhang Y: Role of Jhdm2a in regulating metabolic gene expression and obesity resistance. Nature 2009, 458:757-761.

37. Boily G, Seifert EL, Bevilacqua L, He XH, Sabourin G, Estey C, Moffat C, Crawford S, Saliba S, Jardine K, Xuan J, Evans M, Harper ME, McBurney MW: SirT1 regulates energy metabolism and response to caloric restriction in mice. PLOS ONE 2008, 3:e1759.

38. Torres RMaKR: Laboratory Protocols for Conditional Gene Targeting. Oxford University Press, Oxford, UK 1997.

39. Scherl A, Coute Y, Deon C, Calle A, Kindbeiter K, Sanchez JC, Greco A, Hochstrasser D, Diaz JJ: Functional proteomic analysis of human nucleolus. Mol Biol Cell 2002, 13:4100-4109.

40. Pothlichet J, Mangeney M, Heidmann T: Mobility and integration sites of a murine C57BL/6 melanoma endogenous retrovirus involved in tumor progression in vivo. Int J Cancer 2006, 119:1869-1877.

doi:10.1186/1756-8935-3-8

Cite this article as: Boulard et al:: Histone variant macroH2A1 deletion in mice causes female-specific steatosis. Epigenetics \& Chromatin 2010 3:8.

\section{Submit your next manuscript to BioMed Central and take full advantage of:}

- Convenient online submission

- Thorough peer review

- No space constraints or color figure charges

- Immediate publication on acceptance

- Inclusion in PubMed, CAS, Scopus and Google Scholar

- Research which is freely available for redistribution

Submit your manuscript at www.biomedcentral.com/submit 